\title{
Early immunisation with dendritic cells after allogeneic bone marrow transplantation elicits graft vs tumour reactivity
}

V Gigi ${ }^{1,2,5}$, J Stein ${ }^{1,3,4,5}$, N Askenasy ${ }^{2}$, I Yaniv ${ }^{1,2,3,4,5}$ and S Ash ${ }^{* 1,2,4,5}$

'Zaizov Cancer Immunotherapy Laboratory, Schneider Children's Medical Center of Israel, 14 Kaplan Street, Petach Tikva 49202, Israel; ${ }^{2}$ Frankel Laboratory of Experimental Bone Marrow Transplantation, Schneider Children's Medical Center of Israel, 14 Kaplan Street, Petach Tikva 49202, Israel; ${ }^{3}$ Pediatric Bone Marrow Transplant Unit, Department of Pediatric Hematology-Oncology, Schneider Children's Medical Center of Israel, 14 Kaplan Street, Petach Tikva 49202, Israel; ${ }^{4}$ Department of Pediatric HematologyOncology, Schneider Children's Medical Center of Israel, 14 Kaplan Street, Petach Tikva 49202, Israel and ${ }^{5}$ Sackler Faculty of Medicine, Tel-Aviv University, Tel Aviv, Israel

Background: Perspectives of immunotherapy to cancer mediated by bone marrow transplantation (BMT) in conjunction with dendritic cell (DC)-mediated immune sensitisation have yielded modest success so far. In this study, we assessed the impact of DC on graft vs tumour (GvT) reactions triggered by allogeneic BMT.

Methods: $\mathrm{H}_{2} \mathrm{~K}^{\mathrm{a}}$ mice implanted with congenic subcutaneous Neuro-2a neuroblastoma (NB, $\mathrm{H}_{2} \mathrm{~K}^{\mathrm{a}}$ ) tumours were irradiated and grafted with allogeneic $\mathrm{H}_{2} \mathrm{~K}^{\mathrm{b}}$ bone marrow cells (BMC) followed by immunisation with tumour-inexperienced or tumour-pulsed DC.

Results: Immunisation with tumour-pulsed donor DC after allogeneic BMT suppressed tumour growth through induction of T cellmediated NB cell lysis. Early post-transplant administration of DC was more effective than delayed immunisation, with similar efficacy of DC inoculated into the tumour and intravenously. In addition, tumour inexperienced DC were equally effective as tumour-pulsed DC in suppression of tumour growth. Immunisation of DC did not impact quantitative immune reconstitution, however, it enhanced T-cell maturation as evident from interferon- $\gamma$ (IFN- $\gamma$ ) secretion, proliferation in response to mitogenic stimulation and tumour cell lysis in vitro. Dendritic cells potentiate GvT reactivity both through activation of T cells and specific sensitisation against tumour antigens. We found that during pulsing with tumour lysate DC also elaborate a factor that selectively inhibits lymphocyte proliferation, which is however abolished by humoral and DC-mediated lymphocyte activation.

Conclusion: These data reveal complex involvement of antigen-presenting cells in GvT reactions, suggesting that the limited success in clinical application is not a result of limited efficacy but suboptimal implementation. Although DC can amplify soluble signals from NB lysates that inhibit lymphocyte proliferation, early administration of DC is a dominant factor in suppression of tumour growth.

The antigen-presenting function of dendritic cells (DCs) is a key ingredient bridging between innate and adaptive immunity that has been explored in the context of anti-cancer immunotherapy (Palucka et al, 2007; Kalinski et al, 2009; Kroesen et al, 2012). Functional aspects of antigen-presenting cells (APC) have attracted much interest as potential optimal vehicles of tumour vaccines to achieve selective immune sensitisation against tumours (Berzofsky et al, 2004; Figdor et al, 2004; Rosenberg et al, 2004; Banchereau and Palucka, 2005). The use of DC has shown some efficacy in tumour immunotherapy, yet DC-based vaccines have not fulfilled 
the expected therapeutic benefit (Geiger et al, 2001; Caruso et al, 2005). Despite the apparent correlation between tumour infiltration by DC and the potency of T-cell-mediated anti-tumour reactions (Redlinger et al, 2004), multiple difficulties are yet to be overcome towards successful DC implementation in immunotherapy for NB (Lutz and Schuler, 2002; Raffaghello et al, 2005; Zou, 2005). First, DC have a pivotal role in immune activation but are also endowed with tolerogenic activity that includes induction of anergy in cytotoxic $\mathrm{T}$ cells and activation of regulatory cells (Scheinecker et al, 2002; Cools et al, 2007). Second, DC are submitted to inhibitory signals from malignant cells, such as cytokine-mediated suppression (Sietsma et al, 1998). Third, NB is a particular case of a tumour that impairs DC activity through direct suppression of their development, migration and maturation (Chen et al, 2003; Redlinger et al, 2003; Walker et al, 2005).

Induction of anti-tumour immunity is more effective after bone marrow transplantation (BMT), which eradicates suppressive immune components and provides a chance to re-educate the developing $\mathrm{T}$ cells to attack the malignant tissue (Borrello et al, 2000; Cho et al, 2000). Accordingly, immune activation against immunogenic tumours has been effectively achieved by administration of tumour-pulsed DC after syngeneic BMT (Kapelushnik et al, 1993; Asavaroengchai et al, 2002, 2004), where lymphopenia alleviates the requirement for exogenous administration of immune-stimulatory cytokines. We have demonstrated that allogeneic BMT is significantly more efficient in generating graft vs tumour (GvT) reactions than syngeneic immunohaematopoietic reconstitution, and transition to adaptive immunity after BMT is generally favourable to immune attack of tumours (Ash et al, 2009). Our findings in the murine models of mice bearing congenic Neuro-2a tumours are consistent with clinical evidence underlining superior efficacy of the GvT reaction in limiting tumour growth after allogeneic BMT (Inoue et al, 2003; Li and Waller, 2004; Kanold et al, 2008). Alternatively, induction of immunity has been achieved by allogenisation of tumoural MHC antigens by means of gene transfer (Kobayashi et al, 2007).

In this study, we assessed the involvement of DCs in the evolution of GvT reactivity after allogeneic BMT in a murine model of NB. First, we focused on the optimal timing of DC administration, the impact of donor DC on immune reconstitution and GvT reactivity. We found that early DC administration is beneficial to immune reconstitution, and DC uptake of tumour antigens is effective. Second, we have reported that lymphocytes from tumour bearing mice display proliferative anergy, which is mediated by soluble factors in the tumour lysate (Ash et al, 2009). In view of the tolerogenic activity of DC (Hawiger et al, 2001; Dhodapkar and Steinman, 2002; Liu et al, 2002) we questioned whether these cells abrogate or amplify this lymphocyte-suppressor mechanism. We found that DC intensify both inhibitory and stimulatory signals; however, the dominant effect is suppression of tumour growth through maturation of the reconstituted immune system and tumour antigen-specific sensitisation of cytotoxic T cells.

\section{MATERIALS AND METHODS}

Animal model. Mice used in this study were A/J (H2K $\left.{ }^{\mathrm{a}}, \mathrm{CD} 45.2\right)$, C57BL/6 (H2K $\left.{ }^{\mathrm{b}}, \mathrm{CD} 45.2\right)$ and B6.SJL-Ptprc ${ }^{\mathrm{a}} \mathrm{Pepc}^{\mathrm{b}} / \mathrm{BoyJ}\left(\mathrm{H} 2 \mathrm{~K}^{\mathrm{b}}\right.$, CD45.1) purchased from Jackson Laboratories (Bar Harbor, ME, USA). Mice were housed in a barrier facility in accordance with the guidelines of the Institutional Animal Care and Use Committee.

Tumour cells. Neuro-2a wild-type cells, a mouse NB of strain A origin $\left(\mathrm{H} 2 \mathrm{~K}^{\mathrm{a}}\right)$, were obtained from the American Type Culture Collection (ATCC, Manassas, VA, USA). Cells were cultured to maximum 12 passages in Minimal Essential Medium (Gibco,
Grand Island, NY, USA), supplemented with $10 \%$ fetal bovine serum (FBS), MEM non-essential amino acids, 100 units per ml penicillin, and $100 \mathrm{mg} \mathrm{ml}^{-1}$ streptomycin (Biological Industries, Beit Haemek, Israel). Tumours were induced by subcutaneous (s.c.) implantation of $10^{6}$ Neuro-2a cells suspended in $100 \mu \mathrm{l}$ of phosphate-buffered saline (PBS). Tumour growth was measured with a caliper and the volume $\left(\mathrm{mm}^{3}\right)$ was calculated according to: $\left(\right.$ width $^{2} \times$ length $\left.\times 0.4\right)$. Tumour lysates were prepared by repeated cycles $(\times 3)$ of freezing in liquid nitrogen and thawing of Neuro-2a cells (Ash et al, 2009). Tumours that regressed were excluded from tumour growth curves, to allow separate evaluation of the rates of growth.

Conditioning, transplantation and assessment of reconstitution. Recipients were conditioned with total body irradiation (TBI) at $700 \mathrm{rad}$ using an X-ray irradiator (Rad Source Technologies Inc, Alpharetta, GA, USA) at a rate of $106 \mathrm{rad}$ per min (Kaminitz et al, 2009). After $6 \mathrm{~h}$, cells were injected into the lateral tail vein in $200 \mu \mathrm{l}$ PBS. GVHD was assessed using a semiquantitative clinical scale including weight loss, posture (hyperkeratosis of the foot pads impairs movement), activity, diffuse erythema (particularly of the ear) or dermatitis, and diarrhoea (Ash et al, 2010). Spleen reconstitution was quantified from the total numbers of splenocytes and the fractions of different subsets measured by flow cytometry (Ash et al, 2009).

Cell preparation. Whole BMC (wBMC) were harvested from femurs and tibia of donors, and low-density cells were collected as previously described (Kaminitz et al, 2009). Spleens were harvested from mice, minced, passed through $40 \mu \mathrm{m}$ mesh and dispersed into a single-cell suspension in PBS (Ash et al, 2009). Red blood cells were lysed with medium containing $0.83 \%$ ammonium chloride, $\quad 0.1 \%$ potassium bicarbonate, $0.03 \%$ disodium EDTA (Biological Industries). After $4 \mathrm{~min}$, the reaction was arrested with excess of ice-cold PBS. T cells were enriched by elution through a cotton wool column that preferentially retains $B$ lymphocytes and myeloid cells by differential charge than the eluted $\mathrm{T}$ cells, or immunomagnetic depletion using hybridomaderived antibodies against GR-1, Mac-1 and B220 (Dynal Inc, Lake Success, NY, USA).

Dendritic cells. Mononuclear cells were harvested from bone marrow samples by gradient centrifugation over murine lympholyte (Cedarlane, Burlington, ON, Canada) and cultured $\left(10^{6}\right.$ cells per $\mathrm{ml})$ in low-LPS RPMI-1640 $\left(<10 \mathrm{pg} \mathrm{ml}^{-1}\right)$ supplemented with $10 \%$ FBS, $1 \%$ L-glutamine, $1 \%$ sodium pyruvate, $1 \% \alpha$-MEM non-essential amino acids, $0.1 \%$ Hepes buffer and 1\% Pen/Strep (Biological Industries and Gibco) (Ash et al, 2010). The medium was supplemented with $50 \mathrm{ng} \mathrm{ml}^{-1}$ mouse recombinant (mr)GMCSF and $10 \mathrm{ng} \mathrm{ml}^{-1} \mathrm{mrIL}-4$ on intermittent days (PeproTech, Rocky Hill, NJ, USA). Immunophenotypic characterisation after 6 days of culture showed expression of CD11c $=85 \pm 4 \%$, $\mathrm{CD} 86=65 \pm 6 \%$ and $\mathrm{CD} 205=21 \pm 3 \% \quad(n=5)$. Control DC were incubated for additional $24 \mathrm{~h}$ in DC medium without growth factors. Pulsing with tumour antigens was performed by co-incubation of DC with tumour lysate at an approximate DC:tumour cell ratio of 3:1.

Flow cytometry. Measurements were performed with a Vantage SE flow cytometer (Becton Dickinson, Franklin Lakes, NJ, USA). Positive staining was determined on a log scale, normalised with control cells stained with isotype control mAb. Donor chimerism was determined from the percentage of donor and host peripheral blood lymphocytes (PBL) and splenocytes (Kaminitz et al, 2009). Blood was collected in heparinised serum vials in $200 \mu \mathrm{l} \mathrm{M199,}$ centrifuged over $1.5 \mathrm{ml}$ lymphocyte separation media (Cedarlane), and red blood cells were lysed. Nucleated cells were incubated for $45 \mathrm{~min}$ at $4^{\circ} \mathrm{C}$ with phycoerythrin (PE)-anti-H2 $\mathrm{K}^{\mathrm{b}}$ (Caltag, Carlsbad, CA, USA) and fluorescein isothiocyanate (FITC)-anti- 
$\mathrm{H} 2 \mathrm{~K}^{\mathrm{k}}$ mAb (eBioscience, San Diego, CA, USA), cross reactive against $\mathrm{H}_{2} \mathrm{~K}^{\mathrm{a}}$ (Ash et al, 2009). Minor antigen disparity was assed using CD45.1-PE and CD45.2-FITC antibodies (eBioscience). Lymphocytes were quantified using CD4-allophycocyanin, CD8-FITC antibodies, B220-PerCp and NK1.1-PE and DC were characterised using CD86-PE and CD11c-PerCp antibodies (BD Pharmingen, San Diego, CA, USA).

Lymphocyte functional assays. For measurements of proliferation, cells were labelled with $2.5 \mu \mathrm{M} 5$-(and-6-)-carboxyfluorescein diacetate succinimidyl ester (CFSE, Molecular Probes, Eugene, OR, USA) and plated on petri dishes $\left(5 \times 10^{7}\right)$ for $45 \mathrm{~min}$ to enrich for lymphocytes. After $45 \mathrm{~min}$, non-adherent cells were collected, washed and incubated in DMEM (Ash et al, 2009). Triplicate cultures were harvested after 3-5 days and the dilution of CFSE was analysed by flow cytometry by gating on the live lymphocytes. Data were analysed using the ModFit software (Verity Software House, Topsham, ME, USA).

Interferon- $\gamma$ was determined in supernatant of lymphocyte suspensions in 96-well microtiter plates using the murine IFN- $\gamma$ ELISA kit (BD Pharmingen). Differences absorbance was read in triplicates in an ELISA plate reader (Biotec Inc, Suffolk, UK). Appropriate dilutions within the measurement range were quantified according to a standard calibration curve ( $\left.\mathrm{pg} \mathrm{ml}^{-1}\right)$.

Cytotoxic assays. Effector splenocytes harvested from naïve mice and chimeras were lysed and passed through wool mesh to enrich for $\mathrm{T}$ lymphocytes $(\sim 70 \%)$. These cells were incubated with $5 \times 10^{5}$ Neuro-2a target cells for $7 \mathrm{~h}$ at $37^{\circ} \mathrm{C}$ in $150 \mu \mathrm{l}$ at $1: 10$ to 1:100 target:effector ratios. Cytolysis was quantified by lactate dehydrogenase $(\mathrm{LDH})$ release and normalised for background values (Ash et al, 2009).

Statistical analysis. Data are presented as means \pm standard deviations for each experimental protocol. Results in each experimental group were evaluated for reproducibility by linear regression of duplicate measurements. Differences between the experimental protocols were estimated with a post-hoc Scheffe $t$-test and significance was considered at $P<0.05$.

\section{RESULTS}

Impact of DC administration on tumour growth. In first stage, we assessed the impact of donor DC on graft $v s$ tumour reactivity induced by allogeneic BMT using the experimental setting delineated in Figure 1A: Neuro-2a cells were implanted subcutaneously and after 5 days the mice were irradiated at $700 \mathrm{rad}$ and grafted with allogeneic $\left(\mathrm{H} 2 \mathrm{~K}^{\mathrm{b}} \rightarrow \mathrm{H} 2 \mathrm{~K}^{\mathrm{a}}\right)$ BMC. Dendritic cells $\left(\mathrm{H} 2 \mathrm{~K}^{\mathrm{b}}\right)$ were derived in vitro from donor bone marrow and were pulsed with Neuro-2a lysates $\left(D^{\text {neuro2a }}\right)$ before infusion adjacent to the subcutaneous tumours. Inoculation of $\mathrm{DC}$ on day +7 post transplant caused a significant reduction in tumour growth rates $(P<0.01$ vs allo-BMT; Figure 1B), indicating effective immunisation against the tumour. In this analysis, we excluded tumours that regressed completely to facilitate the statistical analysis of growth rates. Immunisation of DC resulted in complete regression of 3 out of 17 tumours (18\%), whereas only 1 of 21 tumours subsided completely after allogeneic BMT. The allografted mice displayed no signs of GVHD irrespective of DC inoculation, as determined by clinical monitoring and validated by liver histology.

To test whether DC immunisation was mediated by initiation and/or propagation of tumour-reactive T cells, splenic lymphocytes were assessed for direct lysis of Neuro-2a cells in vitro. Lymphocytes from mice immunised with tumour-pulsed DC (DC ${ }^{\text {Neuro2a }}$ ) displayed more potent cytolytic activity as compared with allografted mice $(P<0.005$ at lowest ratio), decreasing the effective target:effector ratio (Figure 1C). These data confirmed that tumour growth suppression is mediated by direct tumour lytic activity of lymphocytes in the allografted mice.

Qualitative effects of DC on immune reconstitution after transplantation. To evaluate the mechanism of action of immunisation with DC, we monitored quantitative and qualitative immune reconstitution at 4 weeks after transplantation. Dendritic cells had little impact on reconstitution of spleen cellularity, which includes delayed recovery of $\mathrm{T}$ cells (Figure $2 \mathrm{~A}$ ). Immaturity of $\mathrm{T}$ cells after allogeneic BMT was recognised from the low output of
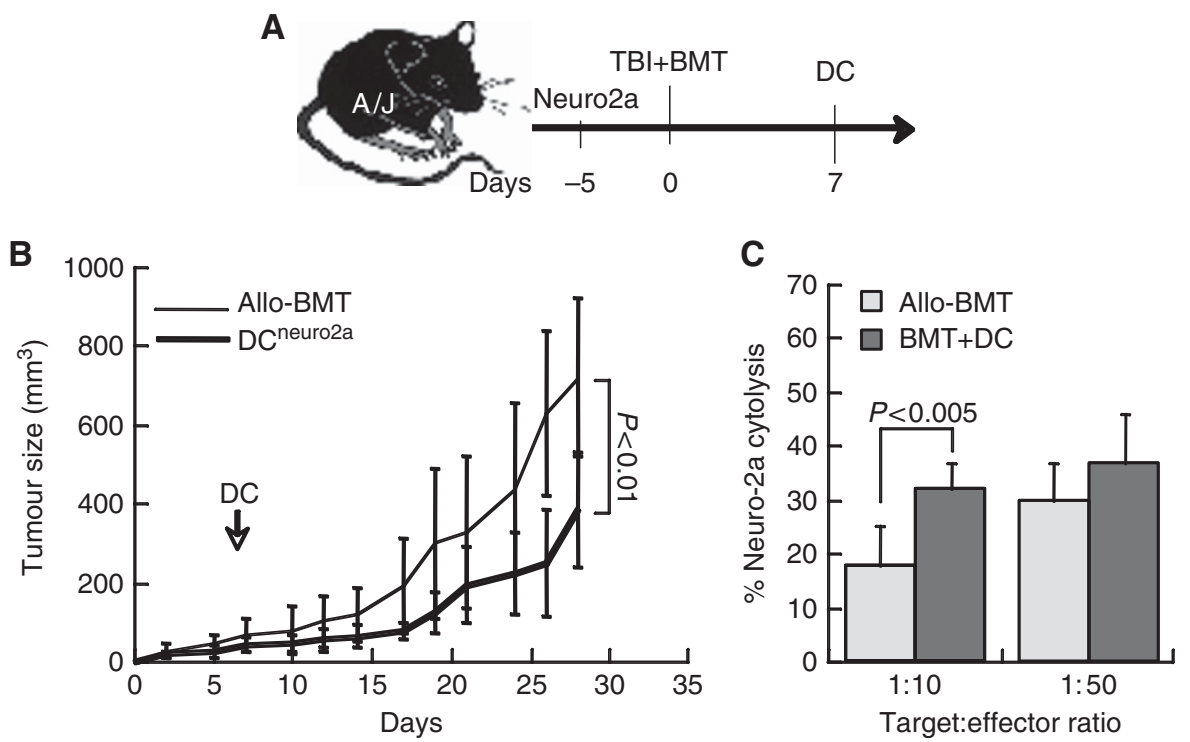

Figure 1. Impact of dendritic cells (DC) on tumour growth and immune reconstitution. (A) The experimental sequence includes subcutaneous implantation of $10^{6}$ Neuro-2a cells (day -5$)$, followed by radiation at 700rad and transplantation of $7 \times 10^{6}$ allogeneic $\mathrm{BMC}\left(\mathrm{H} 2 \mathrm{~K}^{\mathrm{b}} \rightarrow \mathrm{H}_{2} \mathrm{~K}^{\mathrm{a}}\right)$. $10^{6}$ donor DC $\left(\mathrm{H}_{2} \mathrm{~K}^{\mathrm{b}}\right)$ were infused at 7 days post transplantation. (B) Tumour size in allografted mice bearing tumours $(n=21)$ and immunised with tumour-pulsed donor $D C\left(D C^{\text {neuro2a }}, n=14\right)$. (C) Lysis of Neuro-2a cells in vitro as determined from LDH release by effector splenocytes from allografted mice (allo-BMT) and with DC neuro2a immunisation (BMT + DC) at various target:effector ratios. Data represent five mice in each group from one of the repeated experiments. 
interferon- $\gamma$ (IFN- $\gamma)$, which was however somewhat elevated following DC immunisation (Figure 2B). The superior cytokine responses following administration of donor DC prompted more detailed functional analysis of the lymphocytes.

At 4 weeks post transplant, splenic lymphocytes from allografted mice immunised with DC displayed higher proliferative responses under mitogenic stimulation than naïve (unmanipulated) B6 mice and allografted mice $(P<0.01$, Figure $2 \mathrm{C})$. Thus, IFN- $\gamma$ production and proliferation were consistently improved by immunisation with tumour-pulsed donor DC. To further refine the specificity of DC-mediated stimulation, lymphocytes were restimulated in vitro with naïve (antigen-inexperienced) and tumour-pulsed donor DC. Lymphocytes from both control (naïve) and allografted mice were equally stimulated by both types of DC. However, the proliferation of lymphocytes from mice immunised with tumour-pulsed donor DC was further amplified by restimulation with tumour-pulsed DC as compared with tumour-inexperienced DC in vitro $(P<0.05)$, demonstrating enhanced antigen-specific responsiveness of the GvT effectors. These data delineate a scenario where DC participate in maturation of the reconstituted immune system (non-specific mitogenic stimulation), and also in specific sensitisation against tumour cells (restimulation with tumour-pulsed DC).

The most specific tests of cytotoxic GvT effectors are the capacity to lyse tumour cells in vitro. As expected, lymphocytes from allografted mice with and without DC immunisation displayed more potent tumour-lytic activity in vitro than tumour-inexperienced lymphocytes from naïve mice (Figure 2D). However, restimulation with tumour-pulsed DC in vitro elicited more potent tumour lysis than restimulation with naïve DC, pointing to an antigen-specific reaction against the tumour. Therefore, lymphocytes have acquired antigen specificity in allografted mice even in the absence of donor DC infusion, and immunisation with tumour-pulsed donor DC caused only marginal increase in tumour cell lysis. These data suggest that amplification of immune reactivity mediated by DC is more significant than sensitisation to tumour antigens.

Optimal conditions of DC infusion. In view of the beneficial effect on DC immunisation on GvT effectors recovering from lymphopenia following stem cell transplant, we sought to characterise several parameters. We reasoned that delayed infusion of DC after transplantation might be more effective in stimulation of GvT reactivity, under conditions of superior quantitative recovery and maturation of the lymphocytes. This assumption was not substantiated, as early immunisation with tumour-pulsed DC on day +7 was more potent in inhibition of tumour growth than late DC administration on day +14 (Figure $3 \mathrm{~A}$ ). To determine the basis of this differential effect of the time of immunisation, we assessed the proliferative responses of splenic lymphocytes from the immunised mice. Non-specific mitogenic stimulation resulted in higher proliferation rates of lymphocytes from mice immunised on day +7 (Figure 3B), indicating a general activation state. Furthermore, restimulation of the lymphocytes with tumour-pulsed DC in vitro resulted in robust proliferative responses, evidence of tumour-specific sensitisation of lymphocytes in the allografted mice.

These tumour growth curves also emphasise that naïve (antigeninexperienced) DC have similar suppressive effects as tumourpulsed DC at both times of immunisation (Figure 3A). Consistently, lymphocytes from mice immunised with naïve and tumour-pulsed DC showed similar cytolytic activity against Neuro-2a cells in vitro (Figure 3C), indicating effective tumour-specific sensitisation mediated by naïve DC. The effective uptake and presentation of tumour antigens by naïve DC in vivo was affirmed by the robust tumour-lytic activity of lymphocytes upon restimulation with tumour-sensitised DC in vitro. Taken together, these data demonstrate efficient tumour antigen uptake and presentation by naïve donor DC, suggesting that presensitisation to the tumour is not required to induce lymphotoxic anti-tumour reactivity.

The experimental setting used in so far relied on inoculation of the donor DC adjacent to the tumour, however, in the clinical setting DC are required to migrate to and operate at remote sites. We therefore assessed the effect of local vs systemic (intravenous) immunisation with donor DC on tumour growth rates. Tumour suppression was equally affected by DC inoculation adjacent to the tumours and intravenously (Figure 3D), emphasising effective DC migration to distant sites.

Tumour-associated suppressive mechanisms. In a previous study, we reported an interesting decrease in proliferation of lymphocytes from mice bearing tumours exposed to tumour lysate in vitro, suggesting the presence of an inhibitory factor in the soluble fraction of the Neuro-2a extract (Ash et al, 2009). The proliferation of splenocytes from allografted mice immunised with tumour-pulsed DC on day +7 was markedly increased as compared with all other experimental groups, including allografted mice, recipients of $\mathrm{DC}^{\text {naive }}$ and infusion of $\mathrm{DC}$ on day +14 (Figure 4A). To determine whether this inhibitory mechanism is modulated by administration of donor DC, the responses of lymphocytes to mitogenic stimulation were assessed in the presence of tumour lysates. Exposure to Neuro-2a lysate in vitro resulted in suppression of proliferation in one particular group (Figure 4B), suggesting that a tumour-derived inhibitory signal was amplified by immunisation with tumour-pulsed DC early after transplantation. Notably, this effect was restricted to lymphocytes from mice immunised with tumour-pulsed DC and not in lymphocytes of mice immunised with tumour-inexperienced DC. These data suggest that a soluble inhibitory factor was included in the repertoire of antigens processed by DC during pulsing with tumour lysate, and was presented to newly developed $\mathrm{T}$ cells in the allografted mice. However, restimulation of lymphocytes with unstimulated and tumour-pulsed DC ex vivo amplified antigenspecific responses (Figures $2 \mathrm{C}$ and $3 \mathrm{~B}$ ), indicating that this proliferation-inhibitory mechanism does not affect the process of tumour antigen processing and presentation. In addition to reversal of this inhibitory mechanism by restimulation with DC in vitro, proliferative anergy induced by tumour lysate was also reversed by non-specific stimulation of the lymphocytes with LPS in the absence of DC (Figure 4C). Therefore, immune activation overcomes the proliferation-inhibitory signal that is processed by DC during pulsing with tumour antigens.

\section{DISCUSSION}

$\mathrm{T}$ cells are effective mediators of graft $v s \mathrm{NB}$ reactions (Ash et al, 2009, 2010); however, this lineage develops relatively slow and consists of immature cells early after transplantation. A single bolus administration of donor DC elicits potent GvT reactivity of effector lymphocytes that translates into tumour growth inhibition. Immunisation of DC had no significant effect on quantitative immune reconstitution, but improved the function of the reconstituted lymphoid compartment. The salutary effect of immunisation with donor DC is mediated by enhanced maturation of the developing lymphocytes, including non-specific activation as well as tumour antigen-specific sensitisation. The current data indicate that DC should be administered early after transplantation, might be administered either locally or systemically, and immature bone marrow-derived DC do not require pulsing with tumour antigens.

Intravenous and intratumour DC inoculation yielded similar tumour-suppressive activity, demonstrating efficient migration of circulating APC to the tumour. Early immunisation with donor DC (1 week after transplant) improved all functional parameters of the developing $\mathrm{T}$ cells without affecting quantitative recovery of the 

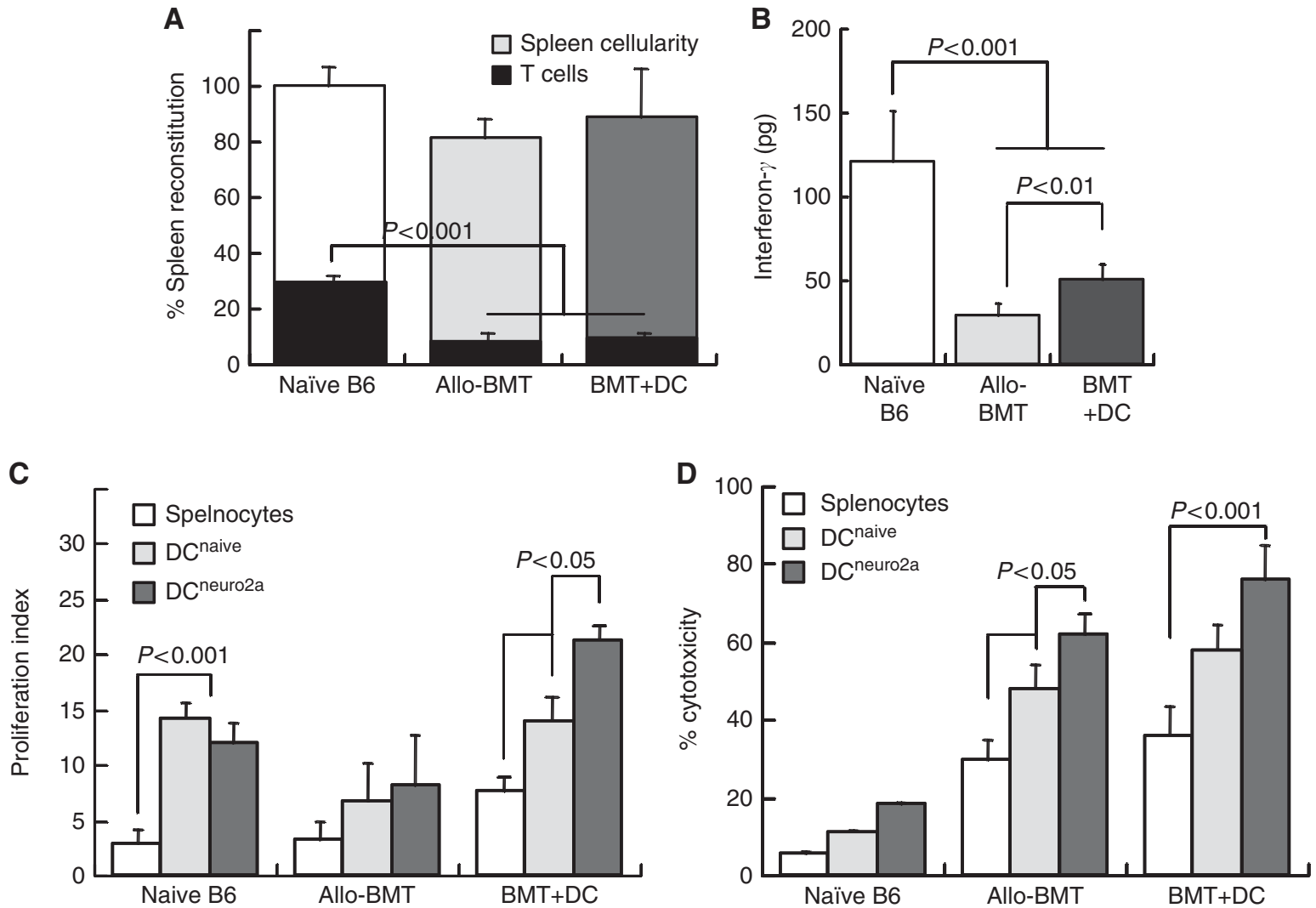

Figure 2. The impact of immunisation with donor DC on quantitative and qualitative immune reconstitution. Comparative analysis of tumourbearing mice undergoing allogeneic bone marrow transplantation (allo-BMT) and with immunisation using donor DC on day + 7 (BMT + DC), as compared with naïve C57BI/6 control mice without any experimental manipulation. Spleens were evaluated at 4 weeks post transplant for: (A) Reconstitution of spleen cellularity and fractions of $\mathrm{CD}^{+}{ }^{+}$and $\mathrm{CD} 8^{+}$T cells at 4 weeks after allogeneic BMT $(n=7)$ and following immunisation with tumour-pulsed donor DC $(n=7)$, as compared with naïve B6 donors $(n=5)$. (B) Interferon- $\gamma($ IFN- $\gamma$ ) secretion in the corresponding experimental groups ( $n=4$ in each group). (C) Proliferation of splenic lymphocytes in response to mitogenic stimulation with ConA as determined from CFSE dilution and quantified with the ModFit software: naïve B6 mice $(n=5)$, allografted mice $(n=8)$ and with DC immunisation $(n=9)$. (D) Neuro-2a cell lysis by effector lymphocytes from spleens of mice in the three experimental groups, and with restimulation in vitro using naïve (antigen-inexperienced, $D^{\text {naive }}$ ) and tumour-pulsed donor DC (DC Neuro2a). Tumour cell lysis was determined from LDH release by splenic lymphocytes at a target:effector ratio of $1: 50$ representing five mice in each group.

lymphoid compartment. Antigen-presenting cells improved IFN- $\gamma$ secretion, responsiveness to mitogen-induced proliferation and direct anti-tumour cytotoxicity, which converged to reduce tumour growth rates. The superior outcome of DC administration in early stages of lymphoid reconstitution suggests that the immunological impact of these cells is primarily mediated by education of the developing donor lymphocytes. Measurements of proliferation and tumour cytotoxicity of lymphocytes from the allografted mice upon restimulation with DC in vitro revealed two activities (Figure 2). The first mechanism involves generalised and nonspecific lymphocyte activation, as revealed by restimulation with naïve DC in vitro. The second mechanism involves selective immunisation against tumour antigens as evident from superior proliferative and cytolytic responses upon restimulation with tumour-pulsed DC in vitro. Tumour antigen-specific immunisation supports our contention that murine NB is immunogenic and is targeted by tumour-specific immune responses after allogeneic BMT (Ash et al, 2009, 2010). Furthermore, our current data confirm a significant role of DC in stimulation of lymphocytes and facilitation of tumour antigen recognition demonstrated by vaccine approaches to induction of anti-tumour immunity using tumour cells modified to express costimulatory molecules and cytokines (Bausero et al, 1996; Johnson et al, 2005) and allogenisation of tumour MHC (Kobayashi et al, 2007).
Naïve DC process antigens following radiation-induced necrosis and apoptosis of tumour cells (Sauter et al, 2000; Tatsuta et al, 2009) and therefore DC interventions are beneficial after irradiation (Tatsuta et al, 2009), syngeneic (Asavaroengchai et al, 2002; Jing et al, 2007) and allogeneic BMT (Ash et al, 2010). It is therefore questioned whether donor DC should be pulsed with tumour antigens before immunisation in the transplant setting. Effective antigen uptake, processing and presentation by naïve (tumour-inexperienced) DC was demonstrated by the similar specific cytolytic responses and tumour growth inhibition as mice treated with tumour-pulsed DC following transplantation (Figure 3C). Efficient uptake of tumour antigens may be explained by the relatively immature nature of DC used in this study, which have superior antigen uptake and presentation capacity as compared with more mature DC (Wilson et al, 2004). We used bone marrow-derived immature DC because unlike mature DC (Lutz and Schuler, 2002), immature cells have reduced capacity to induce regulatory $\mathrm{T}$ cells and to impose anergy on cytotoxic $\mathrm{T}$ cells (Huang et al, 2000). It is therefore apparent that there is no need for pulsing of immature donor DC with tumour antigens for effective post-transplant immunisation. One of the potential drawbacks of DC exposure to tumour lysates is transduction of inhibitory signals derived from the tumour to effector lymphocytes. 

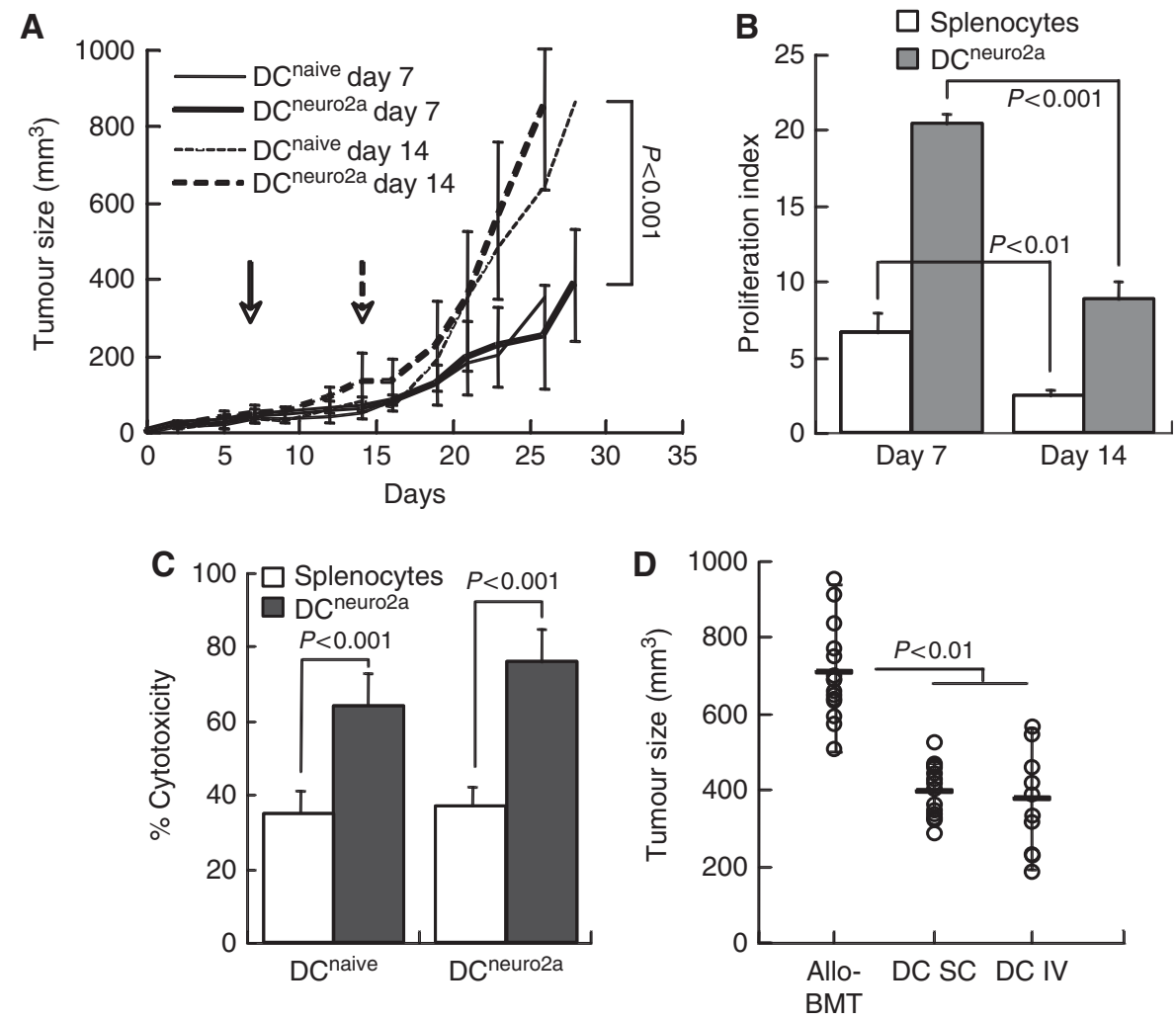

Figure 3. Characteristics of DC immunisation. (A) Tumour growth was monitored in allografted mice immunised on days +7 and +14 (dashed lines) with naïve (DC naive $n=12$ and $n=8$, respectively) and tumour-pulsed DC (DC neuro2a $n=17$ and $n=16$, respectively) as delineated by the arrows. (B) Proliferation of splenic lymphocytes from mice immunised on days $+7(n=9)$ and $+14(n=5)$ with tumour-pulsed DC in response to mitogenic stimulation with ConA, and restimulation with tumour-pulsed DC (DC neuro2a) in vitro. (C) Neuro-2a cell lysis as determined from LDH release by lymphocytes from allografted mice immunised with naïve (antigen-inexperienced) and tumour-pulsed DC on day +7 ( $n=5$ ) and upon restimulation with tumour-pulsed DC in vitro. Assays were performed at a target:effector ratio of 1:50. (D) Tumour size in allografted mice (allo-BMT, $n=21$ ), after subcutaneous (SC, $n=14$ ) and intravenous (IV, $n=11$ ) immunisation with donor DC on day +7 post transplantation.

We have previously reported that lymphocytes from mice bearing tumours display proliferative anergy under mitogenic stimulation (Ash et al, 2009). The same feature of hyporesponsiveness to mitogens was observed in lymphocytes from reconstituted mice immunised with tumour-pulsed DC exposed to tumour lysate. Inhibition pertains primarily to the mitotic function of lymphocytes, as other parameters of maturation, such as IFN- $\gamma$ secretion and tumour cytolysis, were markedly improved by DC therapy. The inhibitory signal revealed here originates from the tumour lysate and is not an intrinsic suppressor activity of DC, because it did not affect lymphocytes of mice inoculated with naïve DC. These data depict a scenario where donor DC engulf and process an inhibitory signal during pulsing with tumour lysate, which is transferred to the emerging lymphocytes along other tumour-specific antigens. Loss of inhibition of proliferation when DC were administered at later times after transplantation suggests that it primarily affects developing $\mathrm{T}$ cells.

Suppression of $\mathrm{T}$-cell proliferation was reversed both by restimulation with DC (Figure 2C) and by non-specific stimulation with LPS (Figure 4C), demonstrating that lymphocyte stimulation overrides the induced proliferative anergy. Current data point to an inhibitory signal that specifically affects effector lymphocyte proliferation, and is distinct from tumour-associated mechanisms capable to inactivate DCs. Prior studies revealed several mechanisms by which NB downregulates GvT reactivity; however, most factors present different characteristics from those observed under our experimental conditions. A possible candidate expressed at high levels by NB is migration inhibitory factor (MIF) that suppresses lymphocyte proliferation; however, work with
MIF-deficient tumours showed that this chemokine is not directly responsible for suppression of $\mathrm{T}$ cell-mediated immunity in vivo (Zhou et al, 2008). Other factors were shown to suppress DC function with variable effect on T cells: IL-27 promotes the evolution of cytotoxic lymphocytes endowed with anti-tumour activity (Shinozaki et al, 2009) and Galectin-1 inhibits cytotoxic T-cell activity (Soldati et al, 2012).

Enhanced GvT activity induced by DC-mediated activation of the reconstituted $\mathrm{T}$-cell subset overcomes specific barriers imposed by neuroblatoma. Stroma of this tumour causes gradual dysfunction of DC (Chen et al, 2003; Redlinger et al, 2003; Walker et al, 2005; Zou, 2005) through downregulation of survival factors and impairment of migration (Kanto et al, 2001; Redlinger et al, 2004). Dysfunction of DC has been effectively reversed by overexpression of proinflammatory cytokines and costimulatory molecules, and downregulation of the sensitivity to suppressor cytokines and chemokines (Zhou et al, 2008; Shinozaki et al, 2009). Additional humoral factors contribute to DC inactivation, for example, high ganglioside-2 (GD2) concentrations in NB patients (McKallip et al, 1999) suppress DC development (Shurin et al, 2001) and function (Sietsma et al, 1998). Repression of anti-tumour immunity by GD2 is attributed to defective cytokine production by DCs (Shurin et al, 2001; Redlinger et al, 2004), which impacts negatively on the development of anti-tumour immunity after syngeneic BMT (Jing et al, 2007).

In summary, DC foster graft $v s \mathrm{NB}$ reactivity through three main mechanisms: facilitation of immune maturation, non-specific activation and induction of antigen-specific GvT reactions. From the clinical point of view, it is important to underline that effective 

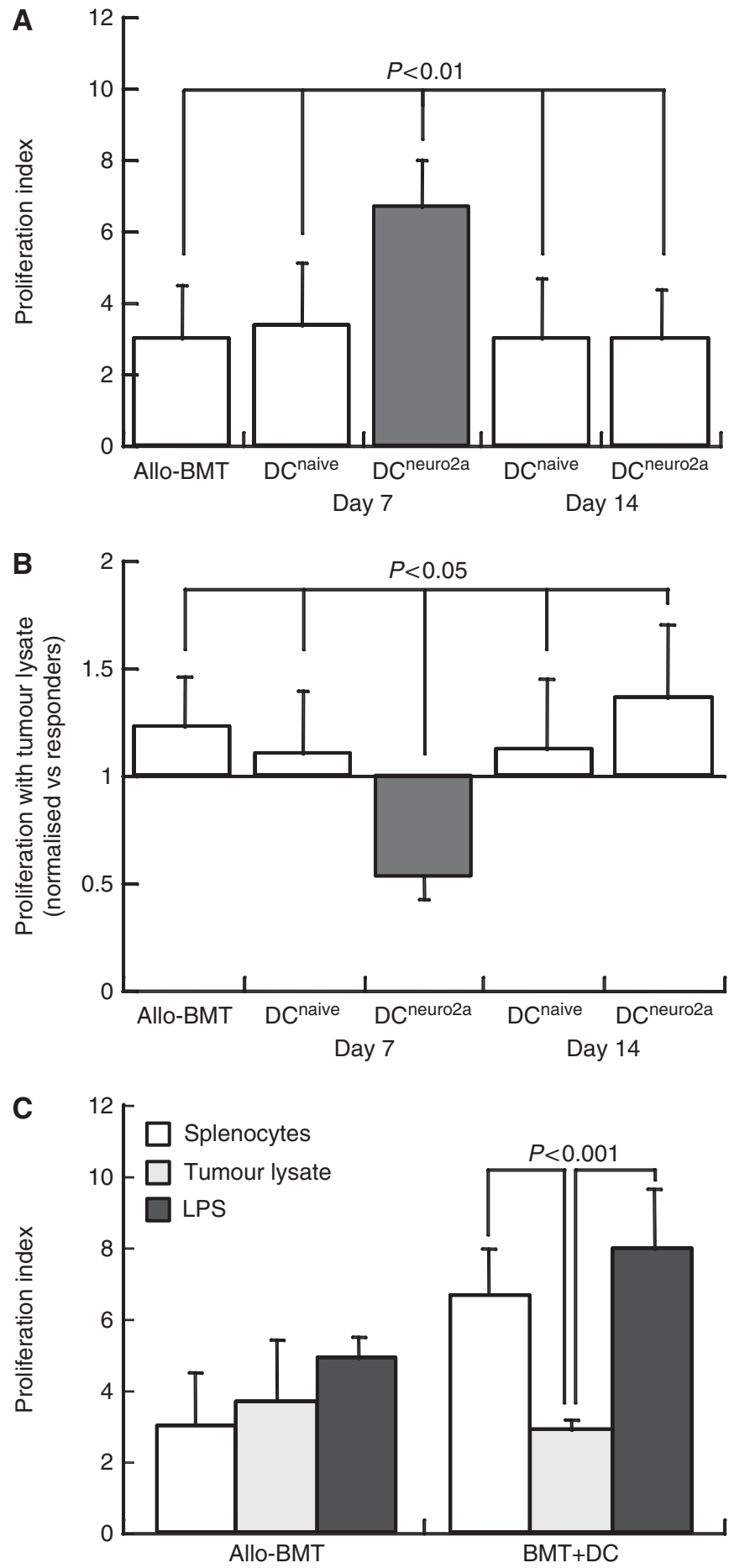

Figure 4. Proliferative responses of lymphocytes. Splenic lymphocytes were harvested at 4 weeks after transplantation and exposed to mitogenic stimulation with ConA in vitro. Proliferation was measured from CFSE dilution. (A) Proliferation rates $(n=5-6)$ of lymphocytes from allografted mice (allo-BMT), and following immunisation with naïve ( $D C^{\text {naive }}$, tumour-inexperienced) and tumourpulsed DC ( $D C^{\text {neuro2a }}$ ) on days +7 and +14 . (B) The impact of tumour lysate on lymphocyte proliferation, expressed as changes normalised against the responses in control medium. (C) Proliferation rates of lymphocytes ( $n=3-5$ in each group) from allografted mice (allo-BMT) and following immunisation with tumour-pulsed DC on day +7 incubated with tumour lysate with and without lipopolysacharide (LPS).
GvT reactivity can be attained already at early stages after transplantation by supplementation of DC, fostering the maturation of bone marrow-derived lymphocytes that are largely devoid of GvT reactivity. Although DC are shown to process and transduce also signals that inhibit lymphocyte proliferation, DC reduce tumour growth and even abrogate established tumours through induction of antigen-specific cytolytic responses.

\section{ACKNOWLEDGEMENTS}

This work was supported by grants from the Israel Cancer Research Fund, Israel Cancer Research Association and the Frankel Trust for Experimental Bone Marrow Transplantation. The excellent technical assistance of Mrs. Ela Zuzovsky and Mrs. Ana Zemlianski is gratefully acknowledged.

REFERENCES

Asavaroengchai W, Kotera Y, Koike N, Pilon-Thomas S, Mulé JJ (2004) Augmentation of antitumor immune responses after adoptive transfer of bone marrow derived from donors immunized with tumor lysate-pulsed dendritic cells. Biol Blood Marrow Transplant 10: 524-533.

Asavaroengchai W, Kotera Y, Mule JJ (2002) Tumour lysate-pulsed dendritic cells can elicit an effective antitumor immune response during early lymphoid recovery. Proc Natl Acad Sci USA 99: 931-936.

Ash S, Gigi V, Askenasy N, Fabian I, Stein J, Yaniv I (2009) Graft versus neuroblastoma reaction is efficiently elicited by allogeneic bone marrow transplantation through cytolytic activity in the absence of GVHD. Cancer Immunol Immunother 58: 2073-2084.

Ash S, Stein J, Askenasy N, Yaniv I (2010) Immunomodulation with dendritic cells and donor lymphocyte infusion converge to induce graft $v s$ neuroblastoma reactions without GVHD after allogeneic bone marrow transplantation. Br J Cancer 103: 1597-1605.

Banchereau J, Palucka AK (2005) Dendritic cells as therapeutic vaccines against cancer. Nat Rev Immunol 5: 296-306.

Bausero MA, Panoskaltsis-Mortari A, Blazar BR, Katsanis E (1996) Effective immunization against neuroblastoma using double-transduced tumor cells secreting GM-CSF and interferon-gamma. J Immunother Emphasis Tumor Immunol 19: 113-124.

Berzofsky JA, Terabe M, Oh S, Belyakov IM, Ahlers JD, Janik JE, Morris JC (2004) Progress on new vaccine strategies for the immunotherapy and prevention of cancer. J Clin Invest 113: 1515-1525.

Borrello I, Sotomayor EM, Rattis FM, Cooke SK, Gu L, Levitsky HI (2000) Sustaining the graft-versus-tumor effect through posttransplant immunization with granulocyte-macrophage colony-stimulating factor (GM-CSF)-producing tumor vaccines. Blood 95: 3011-3019.

Caruso DA, Orme LM, Amor GM, Neale AM, Radcliff FJ, Downie P, Tang ML, Ashley DM (2005) Results of a phase I study utilizing monocytederived dendritic cells pulsed with tumor RNA in children with stage 4 neuroblastoma. Cancer 103: 1280-1291.

Chen X, Doffek K, Sugg SL, Shilyansky J (2003) Neuroblastoma cells inhibit the immunostimulatory function of dendritic cells. J Pediatr Surg 38: 901-905.

Cho BK, Rao VP, Ge Q, Eisen HN, Chen J (2000) Homeostasis-stimulated proliferation drives naive $\mathrm{T}$ cells to differentiate directly into memory T cells. J Exp Med 192: 549-556.

Cools N, Ponsaerts P, Van Tendeloo VFI, Berneman ZN (2007) Balancing between immunity and tolerance: an interplay between dendritic cells, regulatory T cells, and effector T cells. J Leukoc Biol 82: 1365-1374.

Dhodapkar MV, Steinman RM (2002) Antigen-bearing immature dendritic cells induce peptide-specific $\mathrm{CD} 8(+)$ regulatory $\mathrm{T}$ cells in vivo in humans. Blood 100: 174-177.

Figdor CG, De Vries IJ, Lesterhuis WJ, Melief CJ (2004) Dendritic cell immunotherapy: Mapping the way. Nat Med 10: 475-480.

Geiger JD, Hutchinson RJ, Hohenkirk LF, McKenna EA, Yanik GA, Levine JE, Chang AE, Braun TM, Mulé JJ (2001) Vaccination of pediatric solid tumor patients with tumor lysate-pulsed dendritic cells can expand specific $\mathrm{T}$ cells and mediate tumor regression. Cancer Res 61: 8513-8519. 
Hawiger D, Inaba K, Dorset Y, Guo M, Mahnke K, Rivera M, Ravetch JV, Steinman RM, Nussenzweig MC (2001) Dendritic cells induce peripheral $\mathrm{T}$ cell unresponsiveness under steady state conditions in vivo. J Exp Med 194: 769-779.

Huang FP, Platt N, Wykes M, Major JR, Powell TJ, Jenkins CD, MacPherson GG (2000) A discrete subpopulation of dendritic cells transports apoptotic intestinal epithelial cells to T cell areas of mesenteric lymph nodes. J Exp Med 191: 435-444.

Inoue $\mathrm{M}$, Nakano T, Yoneda A, Nishikawa M, Nakayama M, Yumura-Yagi K, Sakata N, Yasui M, Okamura T, Kawa K (2003) Graft-versus-tumor effect in a patient with advanced neuroblastoma who received HLA haplo-identical bone marrow transplantation. Bone Marrow Transplant 32: 103-106.

Jing W, Orentas RJ, Johnson BD (2007) Induction of immunity to neuroblastoma early after syngeneic hematopoietic stem cell transplantation using a novel mouse tumor vaccine. Biol Blood Marrow Transplant 13: 277-292.

Johnson BD, Gershan JA, Natalia N, Zujewski H, Weber JJ, Yan X, Orentas RJ (2005) Neuroblastoma cells transiently transfected to simultaneously express the co-stimulatory molecules CD54, CD80, CD86, and CD137L generate antitumor immunity in mice. J Immunother 28: 449-460.

Kalinski P, Urban J, Narang R, Berk E, Wieckowski E, Muthuswamy R (2009) Dendritic cell-based therapeutic cancer vaccines: what we have and what we need. Future Oncol 5: 379-390.

Kaminitz A, Mizrahi K, Yaniv I, Farkas DL, Stein J, Askenasy N (2009) Low levels of allogeneic but not syngeneic hematopoietic chmerism reverse autoimmune insulitis in prediabetic NOD mice. J Autoimmun 33: 83-91.

Kanold J, Paillard C, Tchirkov A, Merlin E, Marabelle A, Lutz P, Rousseau R, Baldomero H, Deméocq F (2008) Allogeneic or haploidentical HSCT for refractory or relapsed solid tumors in children: toward a neuroblastoma model. Bone Marrow Transplant 42(Suppl 2): S25-S30.

Kanto T, Kalinski P, Hunter OC, Lotze MT, Amoscato AA (2001) Ceramide mediates tumor-induced dendritic cell apoptosis. J Immunol 167: 3773-3784.

Kapelushnik J, Nagler A, Or R, Naparstek E, Cividalli G, Aker M, Mehta J, Mumcuoglu M, Slavin S (1993) Autologous bone marrow transplantation for stage IV neuroblastoma: the role of soybean agglutinin purging. Transplant Proc 25: 2375-2376.

Kobayashi A, Hara H, Ohashi M, Nishimoto T, Yoshida K, Ohkohchi N, Yoshida T, Aoki K (2007) Allogeneic MHC gene transfer enhances an effective antitumor immunity in the early period of autologous hematopoietic stem cell transplantation. Clin Cancer Res 13: 7469-7479.

Kroesen M, Lindau D, Hoogerbrugge P, Adema GJ (2012) Immunocombination therapy for high-risk neuroblastoma. Immunotherapy 4: 163-174.

Li JM, Waller EK (2004) Donor antigen-presenting cells regulate T-cell expansion and antitumor activity after allogeneic bone marrow transplantation. Biol Blood Marrow Transplant 10: 540-551.

Liu K, Iyoda T, Saternus M, Kimura Y, Inaba K, Steinman RM (2002) Immune tolerance after delivery of dying cells to dendritic cells in situ. J Exp Med 196: 1091-1097.

Lutz MB, Schuler G (2002) Immature, semi-mature and fully mature dendritic cells: which signals induce tolerance or immunity. Trends Immunol 23: 445-449.

McKallip R, Li R, Ladisch S (1999) Tumor gangliosides inhibit the tumorspecific immune response. J Immunol 163: 3718-3726.

Palucka AK, Ueno H, Fay JW, Banchereau J (2007) Taming cancer by inducing immunity via dendritic cells. Immunol Rev 220: 129-150.
Raffaghello L, Prigione I, Airoldi I, Camoriano M, Morandi F, Bocca P, Gambini C, Ferrone S, Pistoia V (2005) Mechanisms of immune evasion of human neuroblastoma. Cancer Lett 228: 155-161.

Redlinger RE, Mailliard RB, Barksdale EM (2003) Advanced neuroblastoma impairs dendritic cell function in adoptive immunotherapy. J Pediatr Surg 38: $857-862$.

Redlinger Jr RE, Mailliard RB, Barksdale Jr EM (2004) Neuroblastoma and dendritic cell function. Semin Pediatr Surg 13: 61-71.

Rosenberg SA, Yang JC, Restifo NP (2004) Cancer immunotherapy: moving beyond current vaccines. Nat Med 10: 909-915.

Sauter B, Albert ML, Francisco L, Larsson M, Somersan S, Bhardwaj N (2000) Consequences of cell death: exposure to necrotic tumor cells, but not primary tissue cells or apoptotic cells, induces the maturation of immunostimulatory dendritic cells. J Exp Med 191: 423-434.

Scheinecker C, McHugh R, Shevach EM, Germain RN (2002) Constitutive presentation of a natural tissue autoantigen exclusively by dendritic cells in the draining lymph node. J Exp Med 196: 1079-1090.

Shinozaki Y, Wang S, Miyazaki Y, Miyazaki K, Yamada H, Yoshikai Y Hara H, Yoshida H (2009) Tumor-specific cytotoxic T cell generation and dendritic cell function are differentially regulated by interleukin 27 during development of anti-tumor immunity. Int J Cancer 124: 1372-1378.

Shurin GV, Shurin MR, Bykovskaia S, hogan J, Lotze MT, Barksdale Jr EM (2001) Neuroblastoma-derived gangliosides inhibit dendritic cell generation and function. Cancer Res 61: 363-369.

Sietsma H, Nijhof W, Dontje B, Vellenga E, Kamps WA, Kok JW (1998) Inhibition of hemopoiesis in vitro by neuroblastoma-derived gangliosides. Cancer Res 58: 4840-4844.

Soldati R, Berger E, Zenclussen AC, Jorch G, Lode HN, Salatino M, Rabinovich GA, Fest S (2012) Neuroblastoma triggers an immunoevasive program involving galectin-1-dependent modulation of $\mathrm{T}$ cell and dendritic cell compartments. Int J Cancer 131: 1131-1141.

Tatsuta K, Tanaka S, Tajiri T, Shibata S, Komaru A, Ueda Y, Inoue M, Hasegawa M, Suita S, Sueishi K, Taguchi T, Yonemitsu Y (2009) Complete elimination of established neuroblastoma by synergistic action of gammairradiation and DCs treated with $\mathrm{rSeV}$ expressing interferon-beta gene. Gene Ther 16: 240-251.

Walker SR, Redlinger RE, Barksdale EM (2005) Neuroblastoma-induced inhibition of dendritic cell IL-12 production via abrogation of CD40 expression. J Pediatr Surg 40: 244-250.

Wilson NS, El Sukkari D, Villadangos JA (2004) Dendritic cells constitutively present self-antigens in their immature state in vivo and regulate antigen presentation by controlling the rates of MHC class II synthesis and endocytosis. Blood 103: 2187-2195.

Zhou Q, Yan X, Gershan J, Orentas RJ, Johnson BD (2008) Expression of macrophage migration inhibitory factor by neuroblastoma leads to the inhibition of antitumor T cell reactivity in vivo. J Immunol 181: $1877-1886$.

Zou W (2005) Immunosuppressive networks in the tumor environment and their therapeutic relevance. Nat Rev Cancer 5: 263-274.

This work is published under the standard license to publish agreement. After 12 months the work will become freely available and the license terms will switch to a Creative Commons AttributionNonCommercial-Share Alike 3.0 Unported License. 